\title{
Hiper-hidrose em paciente com traumatismo raquimedular
}

\author{
Carlos Umberto Pereira', Julianne Alves Machado² \\ Serviço de Neurocirurgia do Hospital de Urgência de Sergipe, Aracaju, SE.
}

\section{RESUMO}

A hiper-hidrose é uma condição caracterizada por excessiva sudorese, sobretudo das palmas das mãos e axilas. Pode ser primária ou secundária. A primária tem sido associada com a hiperatividade do sistema nervoso simpático. A hiper-hidrose pode ser tratada clinicamente ou por meio da simpatectomia. Paciente do sexo masculino, 28 anos, ajudante de obras. Vítima de agressão física por arma de fogo. Exame neurológico: desperto. Pupilas isocóricas e ECG 15. Paraplegia crural com nível sensitivo-motor T10. TC da coluna dorsal: fratura do corpo e lâminas de T8, com fragmentos metálicos no canal medular. Quatro meses após o trauma, procurou o ambulatório de Neurocirurgia para acompanhamento do quadro neurológico e referia que após dois meses do trauma apresentou hiper-hidrose axilar e palmar bilateral. Submetido a tratamento conservador, fisioterapia motora e respiratória. Encaminhado ao serviço de Psiquiatria, que referiu a hiper-hidrose como consequência de ansiedade, sendo prescrito antidepressivo tricíclico, mas sem melhora do quadro da hiper-hidrose. Foi encaminhado para tratamento com a dermatologia e a possibilidade de ser submetido à intervenção cirúrgica endoscópica. Até a presente data não retornou ao ambulatório de neurocirurgia. Dentre as complicações clínicas do TRM, a hiper-hidrose tem sido relatada em alguns casos. A produção de suor é afetada após o trauma por causa de uma alteração do sistema nervoso simpático. O tratamento com antidepressivos proporciona apenas alívio parcial e pode apresentar efeitos colaterais. A intervenção cirúrgica, embora passível de efeitos secundários, é um método minimamente invasivo e eficiente no tratamento da hiper-hidrose primária ou secundária.

\section{PALAVRAS-CHAVE}

Traumatismos da medula espinhal, hiper-hidrose, disreflexia autonômica.

\section{ABSTRACT}

Hyperhidrosis in patient with spinal trauma

Hyperhidrosis is a condition characterized by excessive sweating, especially of the hands palms and armpits. It may be primary or secondary. The primary has been associated with hyperactivity of the sympathetic nervous system. Hyperhidrosis can be treated medically or by sympathectomy. Patients, male patient, 28 years old, assistant works. Victim of physical assault by a firearm. Neurological exam: awake. Pupils isochoric and ECG 15. Crural paraplegia with sensory-motor level T10. CT of the spine: fracture of the blades and vertebral body T8, with metal fragments in the spinal canal. Four months after the trauma, came to the neurosurgery clinic for follow-up of the neurological status and stated that two months after the trauma had bilateral palmar and axillary hyperhidrosis. The patient underwent conservative treatment, physical and respiratory therapy. Referred to the Department of Psychiatry, where he was informed that the hyperhidrosis was a consequence of anxiety, and it was prescribed tricyclic antidepressant, but without hyperhidrosis cure. He was referred to dermatology for treatment and the possibility of undergoing surgery as the evolution of endoscopic. To date not returned to the clinic of neurosurgery. Among the clinical complications of SCI, hyperhidrosis has been reported in some cases. The production of sweat is affected after trauma due to a change in the sympathetic nervous system. Treatment with antidepressants provides only partial relief and can have side effects. Surgical intervention, although susceptible to side effects, is a minimally invasive and effective treatment of primary or secondary hyperhidrosis.

\section{KEYWORDS}

Spinal cord injuries, hyperhidrosis, autonomic disreflexia.

1 Professor doutor do Departamento de Medicina da Universidade Federal de Sergipe (UFS); neurocirurgião do Serviço de Neurocirurgia do Hospital de Urgência de Sergipe (HUSE), Aracaju, SE, Brasil.

2 Doutoranda de Medicina da UFS, Aracaju, SE, Brasil. 


\section{Introdução}

Hiper-hidrose $(\mathrm{HH})$ ou sudorese excessiva pode estar presente em pacientes com traumatismo raquimedular (TRM). O padrão de sudorese observado em pacientes com TRM é complexo e não está totalmente esclarecido. Sua fisiopatologia não está bem esclarecida, alguns autores referem que seja devido a uma disfunção do sistema nervoso autonômico (SNA) e à termorregulação em pacientes vítimas de TRM. $\cdot^{1-3}$ O reflexo da sudorese em casos de HH é mediado pelo mesmo mecanismo dos casos em que se desenvolve disreflexia autonômica.

Poucos trabalhos sobre $\mathrm{HH}$ em pacientes com TRM foram publicados. ${ }^{1} \mathrm{~A}$ prevalência de $\mathrm{HH}$ em pacientes com TRM não tem sido estimada na literatura médica. Alguns autores estimam entre $26 \%$ e $29 \%$ dos casos de TRM. ${ }^{2,4}$ Pacientes com TRM cervical são os mais suscetíveis a desenvolverem $\mathrm{HH} .{ }^{3,5}$

A $\mathrm{HH}$ frequente provoca dificuldades e constrangimento no trabalho, vida social e familiar. Dentre as complicações clínicas do TRM, a HH tem sido considerada como uma das mais temíveis devido ao insucesso no seu tratamento. Os autores apresentam um caso de $\mathrm{HH}$ em um paciente com TRM dorsal e discutem sua fisiopatologia e tratamento.

\section{Relato do caso}

Paciente do sexo masculino, 28 anos de idade, ajudante de obras. Vítima de agressão física por arma de fogo. Bom estado geral. Desperto e eupneico. Pupilas isocóricas e escore na escala de coma de Glasgow na admissão 15. Paraplegia crural com nível sensitivo-motor T10. TC de coluna dorsal: fratura do corpo e lâminas da vértebra de $\mathrm{T} 8$, presença de fragmentos metálicos no canal medular. Feito tratamento conservador. Quatro meses após o trauma referia sudorese intensa nas axilas e palmas da mão. Foi encaminhado ao ambulatório de psiquiatria que diagnosticou quadro de ansiedade e depressão, sendo prescrito antidepressivo tricíclico, porém não apresentou melhora. Encaminhado para o ambulatório de dermatologia e orientado a, no caso de insucesso no tratamento clínico, retornar para avaliação de possível tratamento cirúrgico.

\section{Discussão}

A HH pode ser primária (idiopática ou essencial) ou secundária a várias doenças e uso de drogas e pode ser localizada ou generalizada. ${ }^{2}$ As glândulas sudoríparas são inervadas pelo sistema nervoso simpático, sob controle do hipotálamo. Em casos de lesão cervical ou torácica alta, pode interromper as fibras descendentes que formam a cadeia simpática, comprometendo o controle supraespinhal normal. ${ }^{3,6} \mathrm{O}$ reflexo de sudorese que acontece em pacientes com TRM cervical ou torácico alto é devido a algum tipo de estímulo aferente irritativo localizado abaixo do nível da lesão., ${ }^{2,7}$

Uma das causas identificadas de HH pós-TRM inclui a siringomielia pós-traumática ${ }^{8}$, estímulo aferente da bexiga e intestino durante o esvaziamento ou durante $o$ ato de defecação. ${ }^{67}$ A região hiper-hidrótica do corpo corresponde ao nível da lesão na medula espinhal. ${ }^{1} \mathrm{Em}$ pacientes com lesão cervical, a $\mathrm{HH}$ ocorre na cabeça e pescoço (inervado pela cadeia simpática de T1-T4) e ocasionalmente nas extremidades superiores (T2T8). Em pacientes com paraplegia, o tronco (T4-L2) ou membros inferiores (T10-L2) estão afetados. ${ }^{6,9}$ Sua fisiopatologia ainda hoje tem sido controversa, pensando-se que seria pelo mesmo mecanismo do reflexo da sudorese. ${ }^{9}$

A explicação fisiopatológica mais provável é de que em pacientes vítimas de TRM os impulsos aferentes abaixo do nível da lesão entram na medula espinhal via sistema simpático, parassimpático e nervos somatossensoriais através da raiz dorsal do nervo ao longo do trato espinotalâmico ou da coluna dorsal. ${ }^{4}$

Em níveis segmentares até o nível da lesão, os impulsos podem causar um reflexo em massa denominado hiper-reflexia autonômica (ou disreflexia autonômica). Essa hiper-reflexia autonômica foi sugerida para ser o resultado do surgimento de fibras ascendentes, formando sinapses anormais e resultando em uma descarga em massa de neurônios a partir de estímulos periféricos. Eventualmente, essa vazão simpática abaixo do nível da lesão resulta em sinais de hiper-atividade simpática, tais como sudorese, espasmos pilomotores, vasoconstrição no sistema vascular esplânico, na pele ou nas pernas. Devido à falta de inibição supraespinhal à vasoconstrição, como é o caso de pacientes com lesões na medula cervical e torácica alta (acima de T4), pode haver hiper-reflexia autonômica com hipertensão arterial, bradicardia e vasodilatação. ${ }^{4}$

Em pacientes com TRM de níveis de lesão torácica média e inferior, a sudorese é muitas vezes observada abaixo do nível e diminui para baixo. Isso é explicado pelo mesmo mecanismo do TRM cervical. Sudorese acima do nível da lesão em pacientes com lesão medular torácica pode ser explicada pelo mecanismo normal de termorregulação, ativado por um aumento da temperatura central, devido a espasmos pilomotores e vasoconstrição ou por catecolaminas circulantes. A HH em pacientes com lesão lombar é rara, mas tem sido relatada em caso de síndrome da cauda equina. 
O tratamento inicial da $\mathrm{HH}$ em pacientes com TRM envolve inicialmente identificar a causa e remover ou evitar estímulo aferente nóxico (retenção urinária ou fecal), similar ao que se tem realizado para os casos de hiper-reflexia autonômica. ${ }^{5,10} \mathrm{O}$ tratamento da $\mathrm{HH}$ inclui agentes tópicos, medicação sistêmica (gabapentina, propanteline, atropina, antidepressivos, oxibutinina, bloqueadores adrenérgicos, analgésicos narcóticos e drogas anticolinérgicas) e intervenção cirúrgica. ${ }^{11,12}$ Tratamentos alternativos para $\mathrm{HH}$ em pacientes com TRM têm sido realizados por meio da acupuntura, morfina epidural e bloqueio epidural com álcool. ${ }^{13}$ O tratamento sintomático com medicamentos anticolinérgicos e bloqueio simpático no foco na via eferente do arco reflexo e outros alternativos têm sido pouco eficazes ou bem tolerados nesses pacientes. ${ }^{1,11,13,14}$ O tratamento medicamentoso, em muitas ocasiões, não apresenta sucesso ou tem reações medicamentosas adversas; como exemplo, as drogas anticolinérgicas têm, na maioria dos casos, resultados péssimos, e drogas que provocam bloqueio ganglionar e adrenérgico provocam hipotensão postural. ${ }^{4}$ Adams et al. ${ }^{1}$ utilizaram gabapentina com resultado satisfatório, porém o mecanismo de ação dela nesses casos é ainda hoje desconhecido. Esses autores relataram essa droga como tratamento alternativo em pacientes que não responderam a outros medicamentos ou que não toleram os efeitos adversos destes. Staas e Nemunaitis ${ }^{15}$ realizaram aplicação transdérmica de escopolamina em cinco pacientes com $\mathrm{HH}$ e TRM e observaram uma diminuição significativa da sudorese e nenhum efeito adverso dessa droga. Segundo Canaday e Stanford ${ }^{16}$, a droga sistêmica mais utilizada no tratamento de $\mathrm{HH}$ em pacientes com TRM tem sido o brometo de propantelina, um agente anticolinérgico, apresentando resultados satisfatórios.

O tratamento cirúrgico por meio da simpatectomia torácica alta (T2-T3 ou T2-T4) com preservação do gânglio estrelado tem sido usado rotineiramente no tratamento de $\mathrm{HH}$ palmar e axilar. ${ }^{14,17}$ Sudorese compensatória não é rara após simpatectomia torácica alta. ${ }^{18}$

Entre as complicações clínicas do TRM, a HH tem sido relatada. A produção de suor é afetada após o TRM, provavelmente por alterações no SNA. Seu tratamento inicial é medicamentoso e em casos de insucesso pode ser tentado o tratamento cirúrgico com a técnica minimamente invasiva (endoscópica) que consiste na secção cirúrgica de T2 a T4 (simpatectomia), porém esta não está isenta de intercorrências como sudorese secundária em outra localização.

\section{Referências}

1. Adams BB, Vargus-Adams JN, Franz DN, Kinnett DG. Hyperhidrosis in pediatric spinal cord injury: a case report and gabapentin therapy. J Am Acad Dermatol. 2002;46(3):444-6.

2. Gorman $\mathrm{PH}$. Unilateral hyperhidrosis from a contralateral source in an individual with C4 complete tetraplegia. J Spinal Cord Med. 2010;33(4):428-30.

3. Setton ARF, Pereira CU, Santos JL. Hiper-reflexia autonômica simpática. J Bras Neurocirurg. 1995;6(2):49-54.

4. Andersen LS, Biering-Sørensen F, Müller PG, Jensen IL, Aggerbeck $B$. The prevalence of hyperhidrosis in patients with spinal cord injuries and an evaluation of the effect of dextropropoxyphene hydrochloride in therapy. Paraplegia. 1992;30(3):184-91.

5. Kewalramani LS. Autonomic dysreflexia in traumatic myelopathy. Am J Phys Med. 1980;59(1):1-21.

6. Fast A. Reflex sweating in patients with spinal cord injury: a review. Arch Phys Med Rehabil. 1977;58(10):435-7.

7. Haas U, Geng V. Sensation of defecation in patients with spinal cord injury. Spinal Cord. 2008;46(2):107-12.

8. Glasauer FE, Czyrny JJ. Hyperhidrosis as the presenting symptom in post-traumatic syringomyelia. Paraplegia. 1994;32(6):423-9.

9. Head H, Riddoch $\mathrm{G}$. The autonomic bladder, excessive sweating and some other reflex conditions, in gross injuries of the spinal cord. Brain. 1917;40:188-263.

10. Krassioukov A, Warburton DE, Teasell R, Eng JJ. A systematic review of the management of autonomic dysreflexia after spinal cord injury. Arch Phys Med Rehabil. 2009;90(4):682-95.

11. Lefrandt JD, Maurer JM. Oxybutynin for hyperhidrosis. Neth J Med. 2007;65(9):356.

12. Stolman LP. In hyperhidrosis (excess sweating), look for a pattern and cause. Cleve Clin J Med. 2003;70(10):896-8.

13. Yamauchi $\mathrm{Y}$, Kojoh $\mathrm{H}$, Nagaro T, Miyazaki H, Kimura $\mathrm{S}$, Arai T. [Treatment of hyperhidrosis with caudal epidural alcohol block in a patient with cervical cord injury]. Masui. 1993;42(4):606-10.

14. Lin TS. Transthoracic endoscopic sympathectomy for palmar hyperhidrosis in children and adolescents: analysis of 350 cases. J Laparoendosc Adv Surg Tech A. 1999;9(4):331-4.

15. Staas WE Jr, Nemunaitis G. Management of reflex sweating in spinal cord injured patients. Arch Phys Med Rehabil. 1989;70(7):544-6.

16. Canaday BR, Stanford RH. Propantheline bromide in the management of hyperhidrosis associated with spinal cord injury. Ann Pharmacother. 1995;29(5):489-92.

17. Massad MG, Evans A, Rubeiz H, Khoury F, Brown A, Karol $J$, et al. Sympathectomy for truncal hyperhydrosis after traumatic paraplegic injury. J Thorac Cardiovasc Surg. 2002;124(3):636-8.

18. Andrews BT, Rennie JA. Predicting changes in the distribution of sweating following thoracoscopic sympathectomy. $\mathrm{Br} \mathrm{J}$ Surg. 1997;84(12):1702-4.

Endereço para correspondência

Carlos Umberto Pereira

Av. Augusto Maynard, 245/404, São José

49015-380 - Aracaju, SE

E-mail: umberto@infonet.com.br 